\title{
UV color-color relation of early-type galaxies
}

\section{Chang H. Ree ${ }^{1}$, Hyunjin Jeong ${ }^{1}$, Kyuseok $\mathrm{Oh}^{2}$, Chul Chung ${ }^{2}$, Joon Hyeop Lee ${ }^{1}$, Sang Chul Kim ${ }^{1}$ and Jaemann Kyeong ${ }^{1}$}

\author{
${ }^{1}$ Korea Astronomy and Space Science Institute, Daejeon 305-348, Republic of Korea \\ email: chr@kasi.re.kr \\ ${ }^{2}$ Dept. of Astronomy, Yonsei University, Seoul 120-749, Republic of Korea
}

\begin{abstract}
The ultraviolet (UV) color-color relation of early-type galaxies (ETGs) in the nearby universe $(0.05<\mathrm{z}<0.12)$ is re-examined with the latest GALEX GR6 and SDSS DR7 data. By drawing the FUV - NUV (as a measure of UV temperature) versus FUV - $r$ (as a measure of UV amplitude) color-color diagram for the morphologically-cleaned, spectroscopically-cleaned sample of $\sim 3700$ quiescent ETGs, we find that the "old and dead" ETGs consist of a well-defined sequence in UV colors, the "UV red sequence", so that the stronger UV excess galaxies should have a harder UV spectral shape systematically. However, the observed UV spectral slope is too steep to be reproduced by the canonical models in which the UV flux is mainly controlled by age or metallicity parameters. The observed data support the helium enhancement scenario in which the UV spectral shape of UV upturn (FUV - NUV $<0.9$; FUV $-\mathrm{r} \sim 6$ ) galaxies may be governed by the minority population of helium-enhanced horizontal-branch (HB) stars.
\end{abstract}

Keywords. galaxies: elliptical and lenticular, $\mathrm{cD}$, galaxies: evolution, galaxies: stellar content

The $(\mathrm{FUV}-\mathrm{NUV})$ vs. (FUV - r) color-color diagram is a powerful, photometric tool to trace the UV spectral shape and UV amplitude of ETGs, discriminating effectively the contaminations from young stars or AGNs. From the latest GALEX-SDSS matched catalog, we find that the quiescent ETGs in the nearby universe consist of the "UV red sequence" (Ree et al. 2012, ApJ, 744, L10) which was not well identified in previous studies. The observed steep slope of UV color-color relation, (FUV - NUV) $=1.36$ (FUV - r) - 8.35, indicates that the hot HB stars in the UV upturn galaxies should have hotter temperature systematically than those in the UV weak galaxies, at a given UV amplitude. By using two-component model SED syntheses with different assumptions on the UV source populations (young stars, normal or enhanced helium abundances; Fig. 1), we show that the observed UV color distribution is better explained with the additional contribution from minority populations of helium-enhanced HB stars, besides the underlying old populations, as suggested by Chung et al. (2011, ApJ, 740, L45).
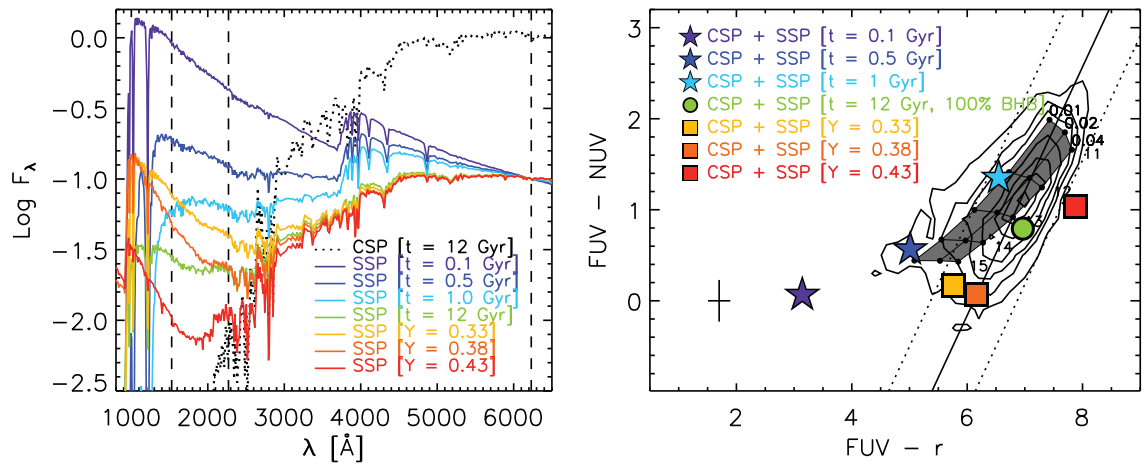

Figure 1. Two-component model SEDs (left) and comparison with the observation (right). 